\title{
Contrast Enhanced Ultrasound Imaging with Nanobubbles: Unique Second-Wave Phenomenon
}

\section{Chuan Chen}

Eindhoven University of Technology

\section{Reshani Perera}

Case Western Reserve University

Michael C. Kolios

Ryerson University

\section{Hessel Wijkstra}

Eindhoven University of Technology

Agata A. Exner

Case Western Reserve University

Massimo Mischi

Eindhoven University of Technology

Simona Turco ( $\nabla$ s.turco@tue.nl)

Eindhoven University of Technology

\section{Research Article}

Keywords:

Posted Date: January 17th, 2022

DOI: https://doi.org/10.21203/rs.3.rs-1247285/v1

License: (c) (i) This work is licensed under a Creative Commons Attribution 4.0 International License.

Read Full License 


\section{Title: Contrast enhanced ultrasound imaging with nanobubbles: unique second-wave phenomenon}

\section{Authors:}

Chuan Chen $^{1}$ (PhD, post-doctoral researcher)

Reshani Perera ${ }^{2}(\mathrm{PhD}$, scientist $)$,

Michael C. $\operatorname{Kolios}^{3}(\mathrm{PhD}$, professor),

Hessel Wijkstra $^{1}(\mathrm{PhD}$, professor),

Agata A. Exner ${ }^{2}(\mathrm{PhD}$, professor),

Massimo Mischi ${ }^{1}(\mathrm{PhD}$, professor),

Simona Turco ${ }^{1 *}(\mathrm{PhD}$, assistant professor, telephone number: +3140247 3821, and e-mail address: s.turco@tue.nl)).

${ }^{1}$ Eindhoven University of Technology, Eindhoven, the Netherlands,

${ }^{2}$ Case Western Reserve University, Cleveland, Ohio, United States,

${ }^{3}$ Ryerson University, Toronto, Canada. 


\section{ABSTRACT}

Investigation of nanobubble (NB) pharmacokinetics in contrast-enhanced ultrasound (CEUS) at the pixel level shows a unique phenomenon where the first pass of the contrast agent bolus is accompanied by a second wave. This effect has not been previously observed in CEUS with microbubbles. The objective of this study was to investigate this second-wave phenomenon and its potential clinical applications. Seven mice with a total of fourteen subcutaneously-implanted tumors were included in the experiments. After injecting a bolus of NBs, the NB-CEUS images were acquired to record the time-intensity curves (TICs) at each pixel. These TICs are fitted to a pharmacokinetic model which we designed to describe the observed second-wave phenomenon. The estimated model parameters are presented as parametric maps to visualize the characteristics of tumor lesions. Histological analysis was also conducted in one mouse to compare the molecular features of tumor tissue with the obtained parametric maps. The second-wave phenomenon is evidently shown in a series of pixel-based TICs extracted from either tumor or tissues. The value of two model parameters, the ratio of the peak intensities of the second over the first wave, and the decay rate of the wash-out process are significantly different between malignant and tissue (Kolmogorov-Smirnov statistic D $>0.25$ ). The occurrence of a second wave is a unique phenomenon that we have observed in NB-CEUS imaging of both mouse tumor and tissue. As the characteristics of the second wave are different between tumor and tissue, this phenomenon has the potential to support the diagnosis of cancerous lesions. 


\section{Introduction}

Among the available ultrasound (US) imaging modes, contrast-enhanced ultrasound (CEUS) is widely used for detection and quantification of blood perfusion $[1,2]$. Following the intravenous administration of ultrasound contrast agents (UCAs) into the blood stream, CEUS allows for higher-sensitivity detection of slow blood flow in the microvasculature as compared to conventional color or power Doppler modes [3]. To this end, dedicated contrast-specific imaging sequences are adopted that drive the MBs close to their resonance frequency and leverage their highly nonlinear response as compared to tissue to enhance the contrast-to-tissue ratio [4]. Currently, the most common UCAs consist of stabilized microbubbles (MBs), where inert gas with low diffusivity is encapsulated in a monolayer phospholipid shell [5]. With a typical diameter of 1-10 $\mu \mathrm{m}$ [5], MBs are intravascular agents. After injecting MBs into the bloodstream, time intensity curves (TIC) can be obtained by measuring the US signal from a local MB concentration as a function of time. The extracted TICs usually exhibit a rapid first pass and relatively weaker recirculation passes of the injected MBs within a time range of 1 minute. Hemodynamic parameters of clinical interest can be extracted from dedicated TIC analysis. The clinical adoption of CEUS has supported and enabled a broad range of applications, ranging from cancer localization [6] to myocardial perfusion [7] up to selective drug delivery by targeted MBs and therapeutic applications [8]. The use of MBs for cancer localization is mostly based on the assessment of perfusion features in the abnormal tumor microvasculature as a result of angiogenesis [6, 9]. Differently, dynamic contrast enhanced (DCE)-MRI and contrast-enhanced CT mainly assess the permeability of angiogenesis, leaky vessels for cancer detection [10]. In fact, the large diameter of MBs, comparable with red blood cells, prevents MB extravasation into the interstitial space, thus hampering their use for assessment of vascular permeability and quantification of biomarkers in the extravascular space [11].

Nanobubbles (NBs) are regarded as a new-generation UCAs whose diameter is more than ten times smaller than conventional MBs $[12,13]$. Despite being off resonance, several studies have demonstrated that NBs can produce high nonlinear contrast enhancement using the standard frequency employed in clinical US [14, 15]. NBs are also proven to be able to extravasate from the vasculature into the interstitial space during their extended life span in the blood circulation $[16,17,18]$. The prolonged retention effect owning to NB extravasation can be used to detect an increase in microvasculature permeability, hence serving as an additional biomarker for detection of cancer angiogenesis [19]. Furthermore, similar to MBs, the NB shell can be decorated to target specific membrane proteins, such as prostate-specific membrane antigen (PSMA), so as to bind to receptors that are overexpressed in cancer cells [20, 21]. Given the diameter of NBs, mostly in the range $100-300 \mathrm{~nm}$, it is reasonable to expect the 
pharmacokinetics of NBs to differ from that of larger intravascular MBs as well as from that of smaller molecular contrast agents commonly used in MRI and CT, such as gadolinium and iodine. In existing studies, the NB pharmacokinetics was indirectly assessed by examining the TIC extracted from a large ROI [14, 16]. From a more general perspective, the analysis of the NB pharmacokinetics can be considered within the category of nanoparticles (NPs) which have similar diameters and reasonably similar properties as NBs [22]. Their small diameter paves avenues for several diagnostic and therapeutic applications, such as cancer localization and drug delivery [23, 24].

In previous studies, the pharmacokinetic analyses of NPs (or NBs) were mostly qualitative, assessed in temporal intervals of hours or days [25], and probed at the organ level [26]. The observed TIC from a large ROI is mostly made up by a first rapidly increasing phase and a second slow decaying phase. By utilizing NBs-based CEUS (NBCEUS), we can achieve both high frame rate and spatial resolution to facilitate the observation NB pharmacokinetics with greater detail. Different from the conventional approach based on TIC assessment from a large ROI [27], analysis of NB-CEUS TICs at the pixel level can bring more insights into the in-vivo pharmacokinetics of NBs.

In this study, we report, to the best of our knowledge for the first time, on the occurrence of a second-wave phenomenon unique to NB pharmacokinetics. The first pass of the UCA bolus is, in fact, accompanied by the appearance of a second wave within a time range of about 15 minutes. This phenomenon has not been observed with conventional MBs. We hypothesized that this second-wave phenomenon is a characteristic of NB pharmacokinetics within tissue. In this study, we focused on the analysis of the second-wave phenomenon observed in NB-CEUS, proposing a model for its description and possible utilization in clinical applications. Our proposed model incorporates the second-wave phenomenon to fit pixel-based TICs extracted from NB CEUS loops.

\section{Materials and Methods}

\section{NB preparation}

In our experiment, we utilized a recently developed NB-UCA whose shell structure is engineered to achieve a higher stability and prolonged half-life during CEUS imaging [28]. For producing this type of NBs, a mixture of glycerol (Gly, Acros Organics) and phosphate buffer saline (PBS) (0.8 mL, Gibco, pH 7.4) was added to a lipid solution (10 $\mathrm{mg} \mathrm{mL}-1$ ). The mixed solution was then sonicated for $10 \mathrm{~min}$ at room temperature and transferred (per $1 \mathrm{~mL}$ ) to a $3 \mathrm{~mL}$ headspace vial. The air inside the vial was manually replaced by octafluoropropane (C3F8, Electronic Fluorocarbons, LLC, PA) gas three times. The phospholipid solution was then activated by mechanical agitation of the vial with a VialMix shaker (Bristol-Myers Squibb Medical Imaging Inc., N. Billerica, MA) for 45 s. The NBs were then isolated from the mixture of bubble population by centrifugation at $50 \mathrm{~g}$ force for 5 minutes. Readers can refer to [28] for more details about the preparation of NBs. In this study, the diameter distribution and concentration 
of NBs were characterized with a Resonant Mass Measurement (RMM, Archimedes, Malvern Instruments) [29]. The diameter of the obtained NBs was measured to be $281 \pm 2 \mathrm{~nm}$. The average concentration of NBs was estimated to be $4.07+/-0.25 \times 1010 \mathrm{NBs}$ per $\mathrm{mL}$.

\section{Animal model}

A total of seven 4-6-weeks old athymic nude mice were employed for acquiring CEUS images. In each mouse, a dual-tumor model, with one PC3flu (PSMA-) tumor on the left side and one PC3pip (PSMA+) tumor on the right side, was initiated by subcutaneously injecting $1 \times 106$ PC3flu and PC3pip cells in $100 \mu \mathrm{L}$ matrigel. Retrovirallytransformed PC3pip cells and transfection-control PC3flu cells were originally obtained from Dr. Michel Sadelain (Memorial-Sloan Kettering Cancer Center, New York, NY) and were obtained for this study from the laboratory of Dr. James Basilion at CWRU. The mice were observed every other day until the tumor's diameter reached 8-10 $\mathrm{mm}$. Before acquiring CEUS imaging, the mice were anesthetized by inhalation of $3 \%$ isoflurane with $1 \mathrm{~L} / \mathrm{min}$ oxygen.

\section{CEUS imaging}

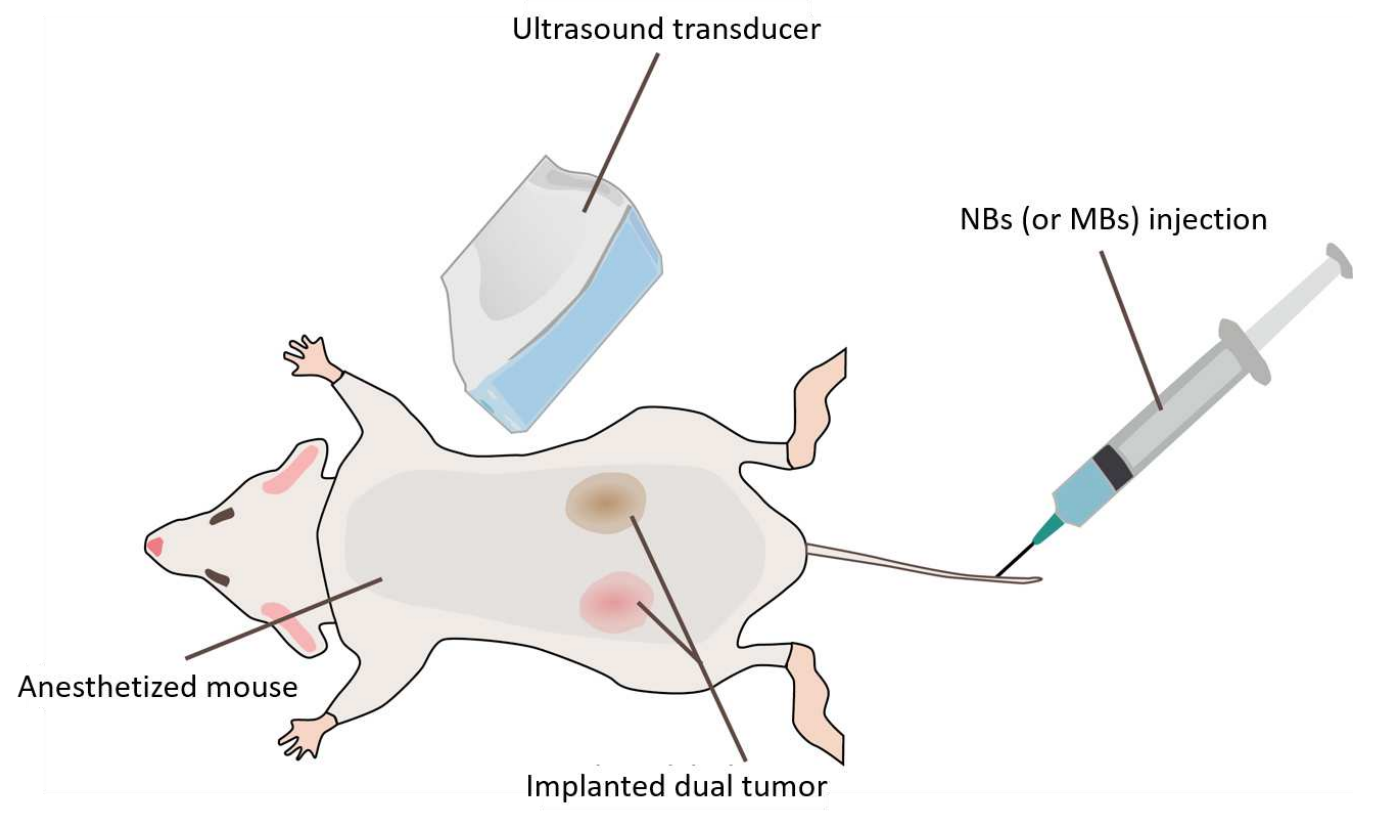

Figure 1: Schematic illustration of the experimental setup. The male athymic nude mice were anesthetized before acquiring CEUS images. A dual tumor was implanted in each mouse subcutaneously in each limb. The ultrasound transducer was fixated at a position at which both tumors were in the field of view. CEUS imaging was performed by administering a $200-\mu \mathrm{L}$ bolus of NBs (or MBs for reference) via the tail vein. 
For acquiring CEUS imaging, 200- $\mu \mathrm{L}$ NBs prepared as above were administrated over $30-40 \mathrm{~s}$ via the tail vein using a $26 \mathrm{G}$ catheter. A PLT-1204BT probe (central frequency, $12 \mathrm{MHz}$; MI, 0.1; dynamic range, $65 \mathrm{~dB}$; gain, 70 $\mathrm{dB}$; imaging frame rate, 0.2 frames/s) connected to a clinical ultrasound machine (AplioXG SSA-790A, Toshiba Medical Imaging Systems) was fixed prior to CEUS imaging to reduce motion artefacts. CEUS acquisitions were performed to image the left PC3flu tumor and the right PC3pip tumor ROI in the same field of view. The CEUS image has a pixel size of $112 \mu \mathrm{m}$. The spatial resolution was estimated to be about $250 \mu \mathrm{m} \times 150 \mu \mathrm{m}$ (lateral $\times$ axial) by measuring the average intensity profiles of several imaging points. Due to storage limitations in the US scanner and to avoid excessive destruction of the contrast agent, the first $5 \mathrm{~min}$ the CEUS loops were recorded at 1 $\mathrm{Hz}$, after which the sampling rate was switched $0.2 \mathrm{~Hz}$. After about 30 minutes, high-intensity flashes were employed to destroy the remaining NBs. The experimental setup of the CEUS acquisition is illustrated in Figure 1. In one mouse, Lumason MBs (sulfur hexafluoride lipid-type A microspheres, Bracco Diagnostics Inc.) were also administrated for acquiring conventional CEUS $30 \mathrm{~min}$ after the NB-CEUS ( $1 \mathrm{~h}$ after first injection, the contrast reached the baseline level), as a reference to be compared with the NB-CEUS.

\section{Data processing and TIC extraction}

After acquiring the NB-CEUS videos, the evolution of the dB-scaled contrast enhancement over time was recorded to obtain a TIC at each pixel. To improve the signal-to-noise ratio, TICs were spatially filtered by convoluting the dB-scaled images with a 2D Gaussian kernel (Full-Width-at-Half-Maximum, FWHM, $300 \mu \mathrm{m}$ ). The choice of this filtering kernel is based on the trade-off between spatial resolution and signal-to-noise ratio. The spatially filtered dB-scaled TICs were then linearized into intensity-scaled TICs by reverting the dynamic-range compression and color mapping implemented in CEUS [30]. A linear relationship between the image intensity and the local concentration of UCAs has been reported in many studies [31]. The TICs were then resampled at $1 \mathrm{~Hz}$ frequency and filtered by a low-pass filter with a passband frequency of $0.1 \mathrm{~Hz}$ for further analysis.

\section{TIC modeling}

The preprocessed, pixel-based TICs were fitted to a new model that incorporates the second-wave phenomenon. This model is fundamentally based on the assumption of a convective-dispersion process of the NBs, as given by the modified local density random walk (mLDRW) model [6], with the addition of a retention compartment, already proposed to describe the transport of targeted MBs [32]. We have extended this model by doubling its parameterization of the convective-dispersion process to also describe the second-wave phenomenon. The resulting model is therefore referred to as double (d)-mLDRW model. By the d-mLDRW model, the local NB concentration, $C(t)$, is considered as the superimposition of two waves, the first pass of the contrast agents and a second wave that 
occurs at least three minutes after UCA administration. Each wave is composed of one intravascular transport function and one additional retention function.

The transport function is described by the mLDRW model as [6]

$$
C_{m L D R W}\left(t ; t_{0}, \kappa, \mu\right)=A U C \sqrt{\frac{\kappa}{2 \pi\left(t-t_{0}\right)}} e^{-\frac{\kappa\left(t-t_{0}-\mu\right)^{2}}{2\left(t-t_{0}\right)}}
$$

where $t_{0}$ is the theoretical injection time, $\mu$ represents the mean transmit time, AUC denotes the area under the curve, and $\kappa$ represents a local dispersion-related parameter given by $\kappa=v^{2} / D$, with $v$ being the NB intravascular velocity and D the NB intravascular dispersion. The retention function is modeled by the convolution between the intravascular function and an exponential function, representing the extravascular compartment [33, 34]. The resulting analytical expression of the d-mLDRW model is

$$
\begin{aligned}
& C(t)=\alpha_{1}\left[C_{m L D R W}\left(t ; t_{0}, \kappa_{1}, \mu_{1}\right)+\beta_{1} C_{m L D R W}\left(t ; t_{0}, \kappa_{1}, \mu_{1}\right) * e^{-\lambda t}\right] \\
& \quad+\alpha_{2}\left[C_{m L D R W}\left(t ; t_{0}, \kappa_{2}, \mu_{2}\right)+\beta_{2} C_{m L D R W}\left(t ; t_{0}, \kappa_{2}, \mu_{2}\right) * e^{-\lambda t}\right] .
\end{aligned}
$$

Here, the function $C_{m L D R W}\left(t ; t_{0}, \kappa, \mu\right)$ represents the mLDRW model that describes the intravascular transport process; $\kappa_{1}$ and $\mu_{1}$ represent the dispersion-related parameter and mean transit time, respectively; $\alpha_{1}$ and $\beta_{1}$ represent intensity scaling ratios of the first wave and the retention function for the first wave, respectively. Likewise, $\kappa_{2}, \mu_{2}, \alpha_{2}$ and $\beta_{2}$ denote the corresponding parameters for the second wave. $\lambda$ represents the decaying rate of retention function. Specifically, we denote the maximum values of the first wave and the second wave as

$$
\begin{aligned}
& \mathrm{m}_{1}=\max \left\{\alpha_{1}\left[C_{m L D R W}\left(t ; t_{0}, \kappa_{1}, \mu_{1}\right)+\beta_{1} C_{m L D R W}\left(t ; t_{0}, \kappa_{1}, \mu_{1}\right) * e^{-\lambda t}\right]\right\}, \\
& \mathrm{m}_{2}=\max \left\{\alpha_{2}\left[C_{m L D R W}\left(t ; t_{0}, \kappa_{2}, \mu_{2}\right)+\beta_{2} C_{m L D R W}\left(t ; t_{0}, \kappa_{2}, \mu_{2}\right) * e^{-\lambda t}\right]\right\},
\end{aligned}
$$

respectively. The time to peak of the first wave and the second wave are represented by $\tau_{1}$ and $\tau_{2}$, respectively.

It can be noticed that the two waves share the same the same theoretical injection time, $t_{0}$, and decaying rate, $\lambda$, for mitigating the risk of overfitting as well as to provide a more realistic representation of the underlying transportretention process. Physiologically, the theoretical UCA injection time, $t_{0}$, is expected to be the same for the two 
waves; the decaying rate $\lambda$ of the retention function is mainly determined by the vascular permeability, which is also expected to be the same for the two waves.

Curve fitting was performed by a least-square fitting method using the trust-region reflective algorithm, as implemented in the Python SciPy package [35]. The initial values, as well as the upper and lower bounds for each parameter, were approximated based on the measured time to peak (TTP), peak enhancement (PE), area under the curve (AUC), and wash-out rate (WoR). Based on our observations, the range of possible $\mu_{1}$ values was bounded between 1 to $2 \mathrm{~min}$, and the range of the possible $\mu_{2}$ values was bounded between 3 to $17 \mathrm{~min}$. For the parameter $t_{0}$, we implemented an iterative search grid spanning from $45 \mathrm{~s}$ to $75 \mathrm{~s}$ in 1 -s steps to search for the smallest squared error. Pixels with a maximum grey level lower than 3 were excluded from the TIC fitting to garantuee adequate levels of signal-to-noise ratio. The goodness of model fitting was evaluated by the coefficient of determination $R^{2}$ defined as:

$$
R^{2}=1-\frac{\left\|C_{e}(t)-C(t)\right\|^{2}}{\left\|C_{e}(t)-\overline{C_{e}}\right\|^{2}}
$$

where $C_{e}(t), C(t)$, and $\|\cdot\|$ represent the measured TIC, the d-mLDRW fitted TIC, and the Euclidean norm, respectively.

\section{Evaluation of the model parameters}

The TIC model fits were evaluated by both quantitative and qualitative assessments. After fitting pixel-based TICs with the proposed d-mLDRW model, parametric maps of $\lambda, \log _{10}\left(m_{2} / m_{1}\right), \tau_{2}$, and $\kappa_{2}$ were produced and visualized. $\lambda$ represents the decaying rate which is affected by NB extravasation and vasculature structure. $\log _{10}\left(m_{2} / m_{1}\right)$ indicates the peak intensity of the second wave relative to the first wave. $\tau_{2}$ and $\kappa_{2}$ represent the characteristics of the second wave in terms of time to peak and convective-dispersion kinetics. The location of the two tumors were roughly delineated by two elliptical-shape ROIs based on both B-mode and CEUS images. The parameter values from the two elliptical-shape tumor ROIs and two neighboring normal-tissue ROIs were compared by the Kolmogorov-Smirnov test to evaluate the parameter ability to distinguish between tumor and tissue. Because the spatial filtering kernel size and spatial resolution are larger than the pixel size, the parametric maps were spatially down-sampled by two times in each direction for increasing the independence of the sample in the statistics. Because the p-value for differentiating large data is usually very low [36], we additionally regarded the KolmogorovSmirnov statistic $D$ to measure the distance between the cumulative probabilities of the parameter distributions for tumor and tissue. The qualitative evaluation was performed by visualization of the parametric maps and a number of pixel TICs, together with the model fit, demonstrating the second-wave phenomenon at the pixel level. 


\section{Histological analysis}

Histological analysis was conducted in one mouse to examine the molecular characteristics of the two tumors. This mouse was also imaged with both NB-CEUS and CEUS with conventional MBs. After the CEUS acquisitions, Phosphate-buffered saline (PBS) perfusion was performed with 50-mL PBS through the left ventricle. After the PBS perfusion, the two tumor lesions were harvested and embedded in the optimal cutting temperature compound (OCT Sakura Finetek USA Inc., Torrance, CA). The tissues were cut into 9- $\mu \mathrm{m}$ slices corresponding to the CEUS acquisition plane, and CD31 staining was performed to visualize the tumor vessels. Briefly, the tissue slices were washed 3 times with PBS and incubated with a protein blocking solution that contain 0.5\% Triton X-100 (Fisher Scientific, Hampton, NH). Tissue slices were incubated in 1:250 diluted CD31 primary antibody (Fisher Scientific, Hampton, $\mathrm{NH}$ ) for $24 \mathrm{~h}$ at $4^{\circ} \mathrm{C}$. After being washed with PBS, the tissue slices were incubated with Alexa 568 tagged secondary antibody (Fisher Scientific, Hampton, NH) for one hour and stained with DAPI (Vecor Laboratories, Burlingame, CA) using standard techniques. Fluorescence images were observed under Leica DM4000B fluorescence microscopy (Leica Microsystem Inc, Buffalo Grove, IL). For comparison with the CEUS images, the fluorescence and histological images were cognitively matched with CEUS images based on the tumor shapes. On the CD31 staining image, one core ROI with about $>1 \mathrm{~mm}$ distance from the tumor border and one rim ROI with about $<1 \mathrm{~mm}$ distance from the tumor border were delineated for each tumor. The average intensities and standard deviation of CD31 staining in the two ROIs were calculated for comparison.

\section{Results}

The model-fitting results of one dual-tumor mouse case are shown in Figure 2. The second-wave phenomenon can be observed in the majority of pixel-based TICs. The parametric maps of both $\log _{10}\left(m_{2} / m_{1}\right)$ and $\lambda$ exhibit a clear difference between the tumor lesion and tissue outside the tumor. The histograms of the parameter values over twelve tumors from six mice (excluding the mouse where MB-CEUS was acquired) are presented in Figure 3. The quantities of measuring the differences between the parameter values, including $\log _{10}\left(m_{2} / m_{1}\right), \lambda, \kappa_{2}$ and $\tau_{2}$, are presented in Table 1. Significantly $\operatorname{lower}_{\log }\left(m_{2} / m_{1}\right)$ values $(\mathrm{D}>0.25)$ and significantly higher $\lambda$ values $(\mathrm{D}>$ 0.25 ) were obtained in tumor lesions compared to the surrounding tissues. For $\lambda$ values there were more than $25.5 \%$ high values $(\lambda>0.6)$ found in tumors in comparison with the $3.3 \%$ high values $(\lambda>0.6)$ presented in tissues. A number of TICs collected from tumor and tissues are presented in Figure 2 to show the second-wave phenomenon at the pixel level. For the total six mice, $86 \%$ of pixel-based TICs were well fitted with the d-mLDRW model with $R^{2}>0.9$. 
Apart from these twelve tumors in six mice, in one mouse both NB-CEUS and MB-CEUS were acquired (see Figure 4). In comparison with MB-CEUS, NB-CEUS produced an overall higher contrast enhancement maintaining a longer period of high contrast enchantment. The region with evident NB perfusion was larger than the region with MB perfusion, especially in the right PC3pip tumor, where NB-CEUS enhancement was high in the peripheral regions and relatively lower in the central regions, while the MBs produced almost no enhancement. In the average TICs collected from two rectangular ROIs of 900 pixels within the two tumors, the presence of a second wave can clearly be recognized in the right ROI (red-colored TIC) and is also visible in the right ROI (blue-colored TIC). For the right PC3pip tumor that presented almost no MB-CEUS enhancement, the estimated $\log _{10}\left(m_{2} / m_{1}\right)$ values in the tumor were not significantly different from the values in tissue. As shown in Figure 4(f), the second wave appears to be stronger for the cyan-colored TIC that is extracted from the right tumor. The second wave can be clearly distinguished from the first wave on the red-colored TIC and blue-colored TIC, both extracted from the rim of the right tumor.

To investigate the biological characteristics and especially the vessel distribution in the two tumors, histological analysis of one mouse case is presented in Figure 5. After cognitive matching of the US images with the histological and fluorescence images, a significant correlation between the location of high CD31 staining and the region of high NB-CEUS enhancement was found in both tumors (see the arrows with corresponding colors). The CD31 staining intensities of the left PC3pip tumor rim and core ROIs were $5.04 \pm 4.88$ and $3.56 \pm 3.84$ (mean \pm standard deviation), respectively. The CD31 staining intensities of the left PC3pip tumor's rim and core ROIs were $5.04 \pm 4.88$ and $3.56 \pm 3.84$ (mean value \pm standard deviation), respectively. The CD31 staining intensities of the right PC3flu tumor rim and core ROIs were $3.71 \pm 6.02$ and $1.38 \pm 3.12$ (mean value \pm standard deviation), respectively.

\begin{tabular}{ccccccc}
\hline \multicolumn{2}{l}{ Table 1. Kolmogorov-Smirnov statistic and p-value for quantifying the differences of parameter values } \\
\hline \\
\hline
\end{tabular}

${ }^{*} \boldsymbol{D}$ represents the Kolmogorov-Smirnov statistic that measures the distance of cumulative probabilities of two distributions 

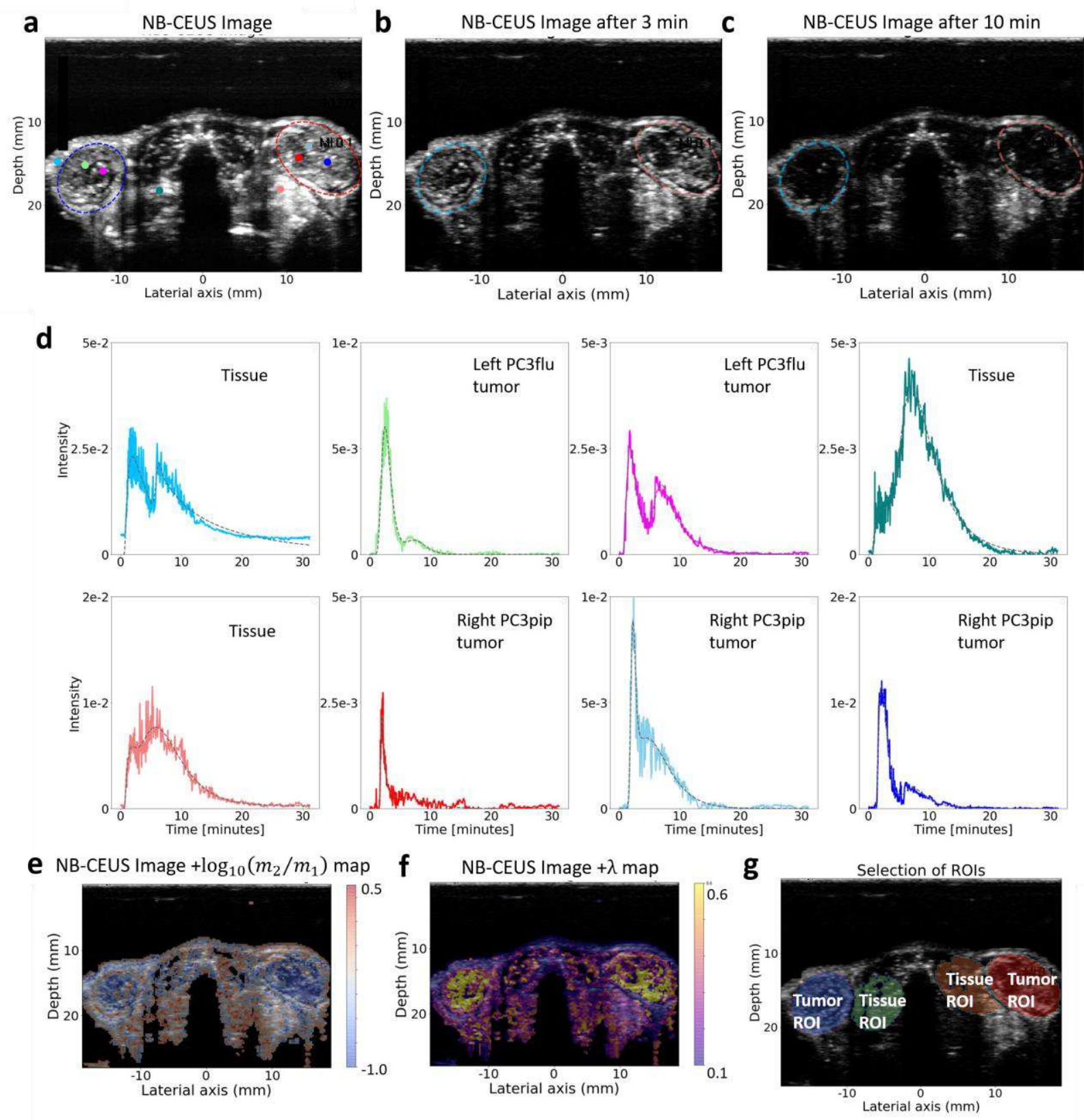

Figure 2: An example of pixel-based TICs and parametric maps in mouse dual-tumor model. (a) presents the maximum intensity projection of the NBs-CEUS loop, with the left PC3flu and right PC3pip tumor ROIs delineated in blue and red, respectively. The NB-CEUS images acquired at time 3 and 15 minutes after the injection are displayed (b) and (c), respectively. In (d), a series of experimental pixel-based TICs (colored solid lines) and corresponding model fit (gray dashed lines) are extracted from different locations which are indicated by the corresponding colored dots in (a). The classification of each dot's location is labelled out. The scales of $y$-axis varies between subfigures in (d). (e) and (f) present the overlays of parametric maps of $\log _{10}\left(m_{2} / m_{1}\right)$ and $\lambda$ upon the NB-CEUS projection image, respectively. In (g) the ROIs corresponding to tumor and tissue are indicated by colored masks. 

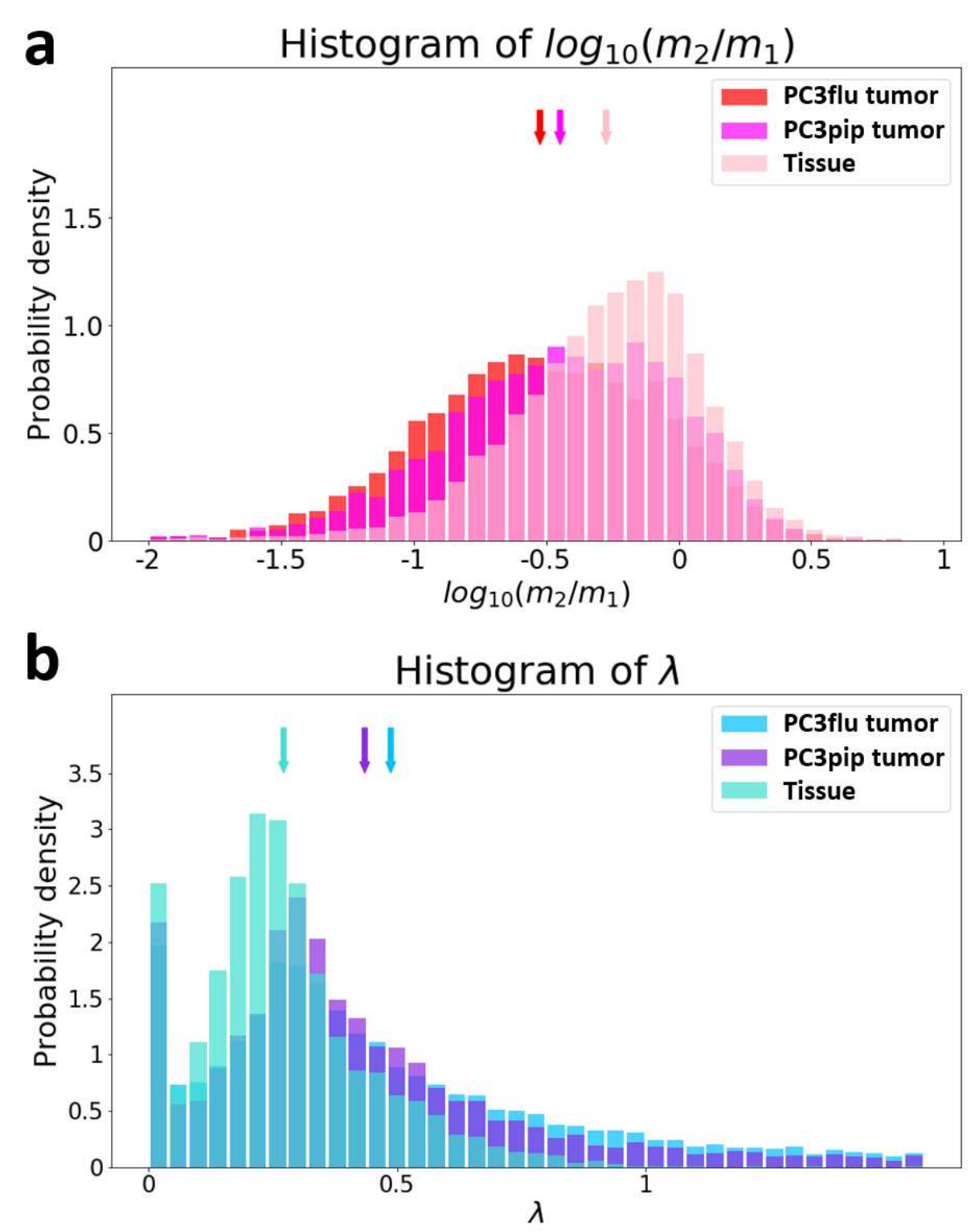

Figure 3: Histogram of parameters $\log _{10}\left(m_{2} / m_{1}\right)$ and $\lambda$ values between the left PC3flu tumor, right PC3pip tumor, and tissue. (a) displays three histograms of $\log _{10}\left(m_{2} / m_{1}\right)$ values collected from six left PC3flu tumor ROIs, six right PC3pip tumor ROls, and twelve reference tissue ROls in six mice. (b) displays three histograms of $\lambda$ values collected from six left PC3flu tumor ROIs, six right PC3pip tumor ROIs, and twelve reference normal-tissue ROIs in six mice. The mean values of these parameters within the two types of tumor ROls and tissue ROls are indicated by arrows of corresponding colors. 

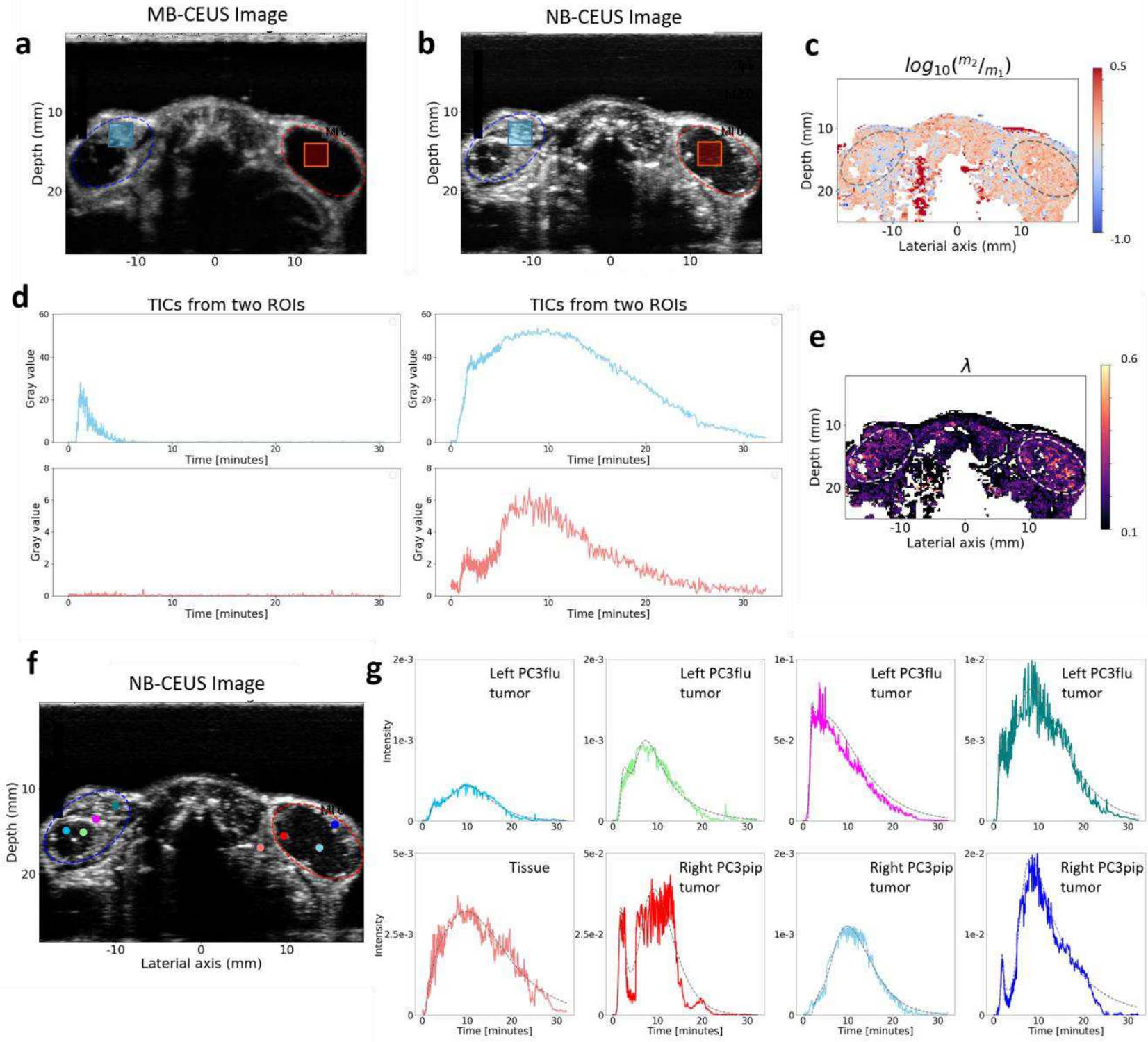

Figure 4: Comparison of parametric maps and TICs obtained on one mouse with MBs and NBs. In the top row, the maximum intensity projection of MB-CEUS (a) and NB-CEUS (b) videos are presented, with the left PC3pip and right PC3 tumor ROls delineated in blue and red, respectively. The average TICs extracted from two rectangular ROIs within the tumors are presented in (d) with corresponding colors. (c) and (e) present the parametric maps of $\log _{10}\left(m_{2} / m_{1}\right)$ and $\lambda$, respectively. In (g), a series of experimental pixel-based TICs (colored solid lines) and corresponding model fit (gray dashed lines) are extracted from the NBs-CEUS video, with the pixels' locations indicated by corresponding colored dots in (f). The scales of the $y$-axis vary between subfigures in (g). 


\section{Discussion}

As an alternative imaging modality to MRI and CT, CEUS is a promising diagnostic tool for enhancing specific organ structures with several advantages, such as low cost, flexibility, wide availability, high spatial and temporal resolution. The development of NBs as a new-generation |UCA can be generally regarded as a special category of NP-based pharmaceuticals and CAs. Partly due to the limitations of existing imaging modalities, previous analyses of the pharmacokinetics of NPs were mostly performed at the organ level, without considering local characteristics of the NP kinetics. In this work we demonstrated the possibility of assessing the TICs in NB-CEUS at the pixel level $(\sim 100 \mu \mathrm{m})$, resulting in the generation of parametric maps. Considering the image spatial resolution and spatial filtering kernel, the resolution of the parametric maps is approximately $300 \mu \mathrm{m}$. In our NB-CEUS experiment in mice, we observed a unique 2nd-wave phenomenon mainly occurring in the time range of 3 to 15 minutes after contrast injection. The overall peak intensity of the second wave often exceeds the intensity of the first wave. To our best knowledge, this second-wave phenomenon has not been described before in the literature describing NPs and NBs. For conventional small-molecular CA in MRI and CT, the shape of pixel-based TICs is typically composed of a the rapidly-rising phase due to CA vascular transport, and a slowly-decaying monotonous phase or plateau due to the extravasation of the contrast agent. In MB-CEUS, a second rise of contrast enhancement due to blood recirculation mainly occurs within 1 minute after the first UCA pass (first wave). However, the intensity of this recirculation wave is generally about 5 times lower than the intensity of the first wave [37]. Given the high intensity (usually exceeding the peak intensity of first wave) and the relatively late occurrence of the second wave (typically reaching the peak between 3 to 15 min after the contrast appearance in the CEUS images), it is unlikely to ascribe the formation of the second wave to intravascular recirculation in the same manner as for MB-CEUS. Instead, the formation of the second wave is more likely to be caused by the in-vivo kinetics of NBs within the internal vasculature and organ structures.

Based on existing pharmacokinetic modeling $[32,34]$, we proposed the d-mLDRW model, by which the variation in NB concentration at the pixel level is described by the superimposition of two waves, both following the mLDRW model extended with an extravascular compartment. By fitting the pixel-based TICs with the proposed d-mLDRW model, we can estimate a number of parameters and visualize them as parametric maps to analyze the different NB pharmacokinetics in tumor and tissue. Our results showed that the estimated $\log _{10}\left(m_{2} / m_{1}\right)$ value is significantly lower in tumors than in tissue. This suggests that a relatively weaker second-wave phenomenon occurs in tumors in comparison to the surrounding tissue. The parametric maps of $\lambda$ showed the decaying rate of the NB concentration to be overall more prominent in the tumor than in the surrounding tissue. These differences can be 


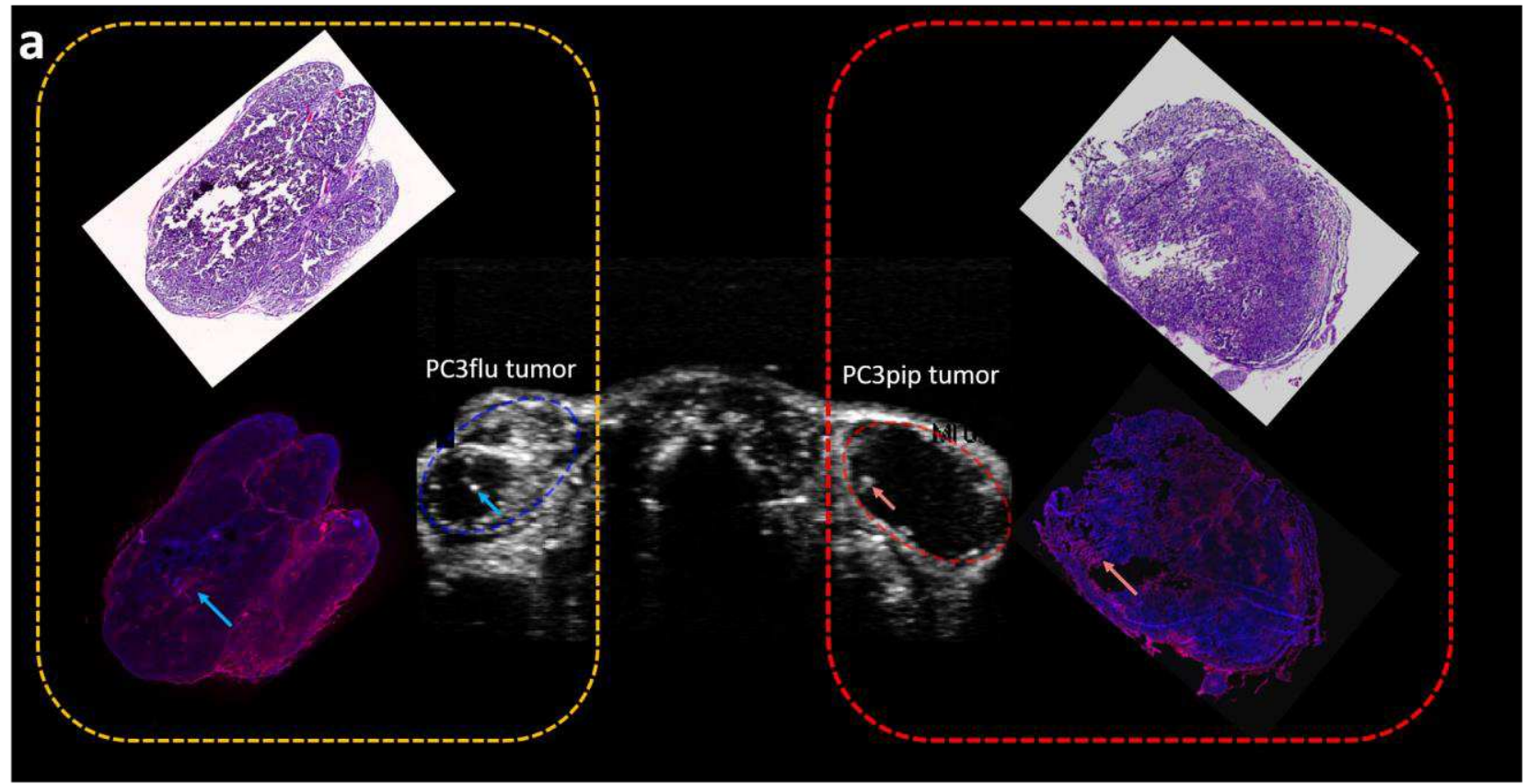

b

DAPI

CD31

C

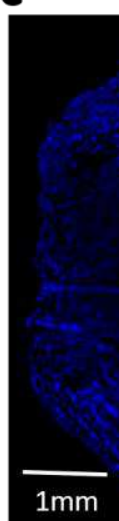

DAPI

CD31

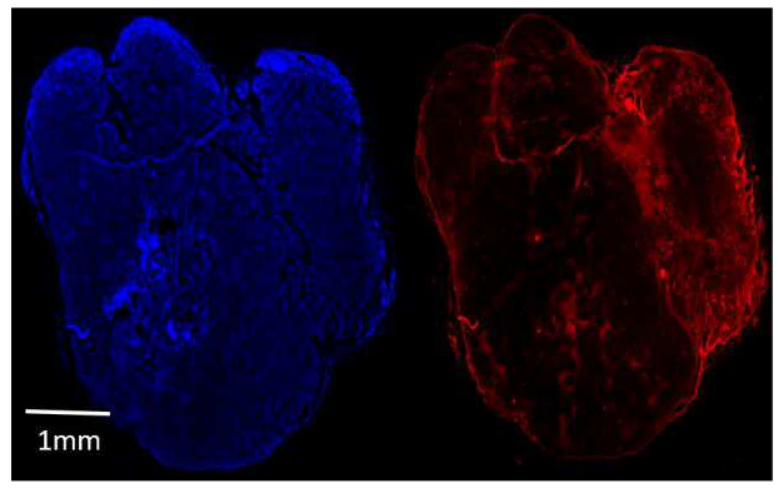

Figure 5: Histology and fluorescence imaging analyses of the dual-tumor model in one mouse. In (a), the rotated histology images and fluorescence images that overlay aligned DAPI and CD31 staining images are presented by the sides of corresponding left PC3pip and right PC3flu tumors, Two pairs of colored arrows indicate the correlation between the location of high CD31 staining and the region of high NB-CEUS enhancement. The orientations of histology images and fluorescence images are based on the shape of tumors. The separate DAPI and CD31 fluorescence images of the left PC3pip and right PC3flu tumors are presented in (b) and (c), respectively. 
probably ascribed to the abnormal tumor vasculature caused by angiogenesis. Different from the normal vasculature that exhibits a regular branching with typical arteriole-capillary-venule hierarchy, the vasculature in the tumor usually lacks this hierarchical structure and presents irregular and chaotic branching. To support tumor growth, tumor angiogenesis yields a vascular network with large functional blood vessels, higher microvascular density, tortuous morphology, excessive branching, abnormal shunts, and increased permeability [38]. The larger functional blood vessels and abnormal shunts could guide more NBs to flow through large vessels and circumvent the slower perfusion in the capillary bed. The high microvascular density, blood shunting, and excessive branching can probably facilitate the washout process of NBs. A faster washout process in tumor in comparison to tissues was also observed in studying the in-vivo kinetics of MBs [6]. The combination of these abnormal features can possibly contribute to the observed relatively weaker second wave, and a faster wash-out process in tumors. The other factors, e.g. NBs extravasation from the leaky vessels, likely also play roles in altering the characteristics of the secondwave phenomenon.

With regard to the right tumor in the exceptional case with almost no MB enhancement and low CD31 staining intensities, we hypothesize that this tumor presented low vessel density. This can serve as a possible explanation for the lack of MB perfusion in this tumor. Probably due to the smaller diameter, NBs can still efficiently perfuse the tumor rim and penetrate the tumor core. The absence of MBs and low perfusion of NBs in this PC3pip tumor may possibly be explained by the increased resistance to blood flow in the case of tumor hypoxia [39]. The increased resistance makes particle transport into the tumor core difficult.

As shown in the parametric maps of $\log _{10}\left(m_{2} / m_{1}\right)$, the regions of relatively higher-intensity first wave were mostly correlated with the regions of high contrast enhancement; the regions of relatively higher-intensity second wave mostly overlap with the regions of low contrast enhancement. Based on common knowledge of MB-CEUS, high contrast enhancement usually occurs in large vessels, while low contrast enhancement indicates the absence of large vessels. Hence, we can hypothesize that the second wave mainly represents NB perfusion of the capillary bed and the interstitial spaces; instead, the first wave mainly represents the NB transport in large vessels. As one imaging pixel might contain both large vessels, small capillaries, and interstitial spaces, the TIC of one imaging pixel can be interpreted as the overlay of the fast transport of NBs in large vessels and the slower perfusion in the small capillary bed or interstitial spaces. In the presence of blood shunts in the tumor, the fast transport and slower perfusion of NBs will be gathered in the veins resulting in two well separated waves. This is one interpretation that can explain the clear separation of two waves in the TICs from the hyper-enhancing rim regions of the right PC3pip tumor in Figure 3. We hypothesize that the second-wave phenomenon is a combination of the fast NB transport in large vessels and the slow NB perfusion of the capillaries and interstitial spaces, probably also involving the role of blood shunts. In the future, dedicated pharmacokinetic modeling will be developed to provide a more accurate 
description of the NB pharmacokinetics in large vessels, capillary bed, and interstitial spaces to verify our hypothesis.

Two parameters, e.g., the relative intensities of two waves $\log _{10}\left(m_{2} / m_{1}\right)$ and the decaying rate $\lambda$, were significantly different between tumors and tissues. Although not presenting significant differences as $\log _{10}\left(m_{2} / m_{1}\right)$ and $\lambda$, the value of other parameters, such as the skewness $\kappa_{2}$ and time delay $\tau_{2}$ of the second wave, are also worthwhile to be further investigated by improving the quantitative fitting model and physiology analyses. It is also noted that the result in this study is still preliminary and limited to seven mice. The validity of the developed d-mLDRW model needs to be further evaluated and optimized. Furthermore, this study only included the histological analysis of one mouse as reference. In future studies, more histological data should be acquired to provide additional evidence of the correlation between the features of second-wave phenomenon and the underlying (immuno)histopathological tissue and vascular characteristics. With regard to the newly observed second-wave phenomenon, there are still several aspects that need further investigation. Primarily, the mechanism underlying the second-wave generation needs to be further researched via both theoretical modeling and experimental validation. Secondly, the roles of several possible factors, e.g., the NBs extravasation, distribution of large vessels, the microstructure of the capillary, and NB diameter, in affecting the second-wave phenomenon requires thorough investigation. Thirdly, it is of value to consider the characteristics of the second-wave phenomenon presented in various organ structures, including different types of tumors, kidneys, liver, and spleen. Lastly, it remains unclear if the second-wave phenomenon observed in NB-CEUS also applies to the other types of NPs. Briefly, the secondwave phenomenon suggests that the characteristics and potential applications of NBs are expanded compared to those of current MBs.

To conclude, in this study, we presented preliminary results to reveal the unique second-wave phenomenon observed in NB-CEUS. By pharmacokinetic modeling that incorporates the second wave, a number of parametric maps were obtained to analyze the different pharmacokinetics of NBs in tumor and tissues. We obtained significantly different parameter values between tumors and tissues, suggesting the potential application of NBs in cancer diagnostics. The applications, mechanism, and detailed characteristics of the second-wave phenomenon are topics of further investigation.

\section{Ethical approval}


Mice were handled in accordance with ARRIVE guidelines and approved by the Institutional Animal Care and Use Committee (IACUC) at Case Western Reserve University (CWRU) in accordance with all applicable protocols and guidelines.

\section{Data availability}

The datasets used and/or analyzed during the current study available from the corresponding author on reasonable request. 


\section{References}

1. D. Maulik, I. Zalud, Doppler ultrasound in obstetrics and gynecology. (Springer, 2005).

2. M. Claudon et al., Guidelines and good clinical practice recommendations for contrast enhanced ultrasound (CEUS)-update 2008. Ultraschall in der Medizin-European Journal of Ultrasound 29, 28-44 (2008).

3. M. Krix et al., Comparison of intermittent-bolus contrast imaging with conventional power Doppler sonography: quantification of tumour perfusion in small animals. Ultrasound in medicine \& biology 29, 10931103 (2003).

4. P. J. Frinking, A. Bouakaz, J. Kirkhorn, F. J. Ten Cate, N. De Jong, Ultrasound contrast imaging: current and new potential methods. Ultrasound in medicine \& biology 26, 965-975 (2000).

5. J.-M. Gorce, M. Arditi, M. Schneider, Influence of bubble size distribution on the echogenicity of ultrasound contrast agents: A study of SonoVue ${ }^{\mathrm{TM}}$. Investigative radiology 35, 661-671 (2000).

6. M. P. Kuenen, M. Mischi, H. Wijkstra, Contrast-ultrasound diffusion imaging for localization of prostate cancer. IEEE transactions on medical imaging 30, 1493-1502 (2011).

7. K. Wei et al., Quantification of myocardial blood flow with ultrasound-induced destruction of microbubbles administered as a constant venous infusion. Circulation 97, 473-483 (1998).

8. K. Kooiman, H. J. Vos, M. Versluis, N. de Jong, Acoustic behavior of microbubbles and implications for drug delivery. Advanced drug delivery reviews 72, 28-48 (2014).

9. M. Kuenen, T. Saidov, H. Wijkstra, M. Mischi, Contrast-ultrasound dispersion imaging for prostate cancer localization by improved spatiotemporal similarity analysis. Ultrasound in medicine \& biology 39, 16311641 (2013).

10. C. Cuenod, D. Balvay, Perfusion and vascular permeability: basic concepts and measurement in DCE-CT and DCE-MRI. Diagnostic and interventional imaging 94, 1187-1204 (2013).

11. M. Smeenge et al., First-in-human ultrasound molecular imaging with a VEGFR2-specific ultrasound molecular contrast agent (BR55) in prostate cancer: a safety and feasibility pilot study. Investigative radiology 52, 419-427 (2017).

12. T. Yin et al., Nanobubbles for enhanced ultrasound imaging of tumors. International journal of nanomedicine 7, 895 (2012).

13. R. H. Perera et al., Ultrasound imaging beyond the vasculature with new generation contrast agents. Wiley Interdisciplinary Reviews: Nanomedicine and Nanobiotechnology 7, 593-608 (2015).

14. $\quad \mathrm{H}$. Wu et al., Acoustic characterization and pharmacokinetic analyses of new nanobubble ultrasound contrast agents. Ultrasound in medicine \& biology 39, 2137-2146 (2013).

15. A. A. Exner, M. C. Kolios, Bursting Microbubbles: How Nanobubble Contrast Agents Can Enable the Future of Medical Ultrasound Molecular Imaging and Image-Guided Therapy. Current Opinion in Colloid \& Interface Science, 101463 (2021).

16. T. Yin et al., Nanobubbles for enhanced ultrasound imaging of tumors. International journal of nanomedicine 7, 895 (2012).

17. C. Pellow, E. C. Abenojar, A. A. Exner, G. Zheng, D. E. Goertz, Concurrent visual and acoustic tracking of passive and active delivery of nanobubbles to tumors. Theranostics 10, 11690 (2020).

18. H. Wu, E. C. Abenojar, R. Perera, T. An, A. A. Exner, Time-intensity-curve analysis and tumor extravasation of nanobubble ultrasound contrast agents. Ultrasound in medicine \& biology 45, 2502-2514 (2019).

19. D. G. Ramirez et al., Contrast-enhanced ultrasound with sub-micron sized contrast agents detects insulitis in mouse models of type1 diabetes. Nature communications 11, 1-13 (2020).

20. R. H. Perera et al., Real time ultrasound molecular imaging of prostate cancer with PSMA-targeted nanobubbles. Nanomedicine: Nanotechnology, Biology and Medicine 28, 102213 (2020).

21. Y. Wang et al., Molecular imaging of orthotopic prostate cancer with nanobubble ultrasound contrast agents targeted to PSMA. Scientific Reports 11, 1-12 (2021).

22. M. De, P. S. Ghosh, V. M. Rotello, Applications of nanoparticles in biology. Advanced Materials 20, 4225-4241 (2008). 
23. E. Blanco, H. Shen, M. Ferrari, Principles of nanoparticle design for overcoming biological barriers to drug delivery. Nature biotechnology 33, 941-951 (2015).

24. D. P. Cormode, P. C. Naha, Z. A. Fayad, Nanoparticle contrast agents for computed tomography: a focus on micelles. Contrast media \& molecular imaging 9, 37-52 (2014).

25. B. Du, M. Yu, J. Zheng, Transport and interactions of nanoparticles in the kidneys. Nature Reviews Materials 3, 358-374 (2018).

26. P. Dogra et al., Mathematical modeling in cancer nanomedicine: a review. Biomedical Microdevices 21, $1-23$ (2019).

27. H. Wu, E. C. Abenojar, R. Perera, T. An, A. A. Exner, Time-intensity-curve analysis and tumor extravasation of nanobubble ultrasound contrast agents. Ultrasound in medicine \& biology 45, 2502-2514 (2019).

28. R. Perera et al., Contrast enhanced ultrasound imaging by nature-inspired ultrastable echogenic nanobubbles. Nanoscale 11, 15647-15658 (2019).

29. C. Hernandez et al., Sink or float? Characterization of shell-stabilized bulk nanobubbles using a resonant mass measurement technique. Nanoscale 11, 851-855 (2019).

30. S. Turco, I. Tardy, P. Frinking, H. Wijkstra, M. Mischi, Quantitative ultrasound molecular imaging by modeling the binding kinetics of targeted contrast agent. Physics in Medicine \& Biology 62, 2449 (2017).

31. M. Arditi, P. J. Frinking, X. Zhou, N. G. Rognin, A new formalism for the quantification of tissue perfusion by the destruction-replenishment method in contrast ultrasound imaging. IEEE transactions on ultrasonics, ferroelectrics, and frequency control 53, 1118-1129 (2006).

32. S. Turco et al., Contrast-enhanced ultrasound quantification: from kinetic modeling to machine learning. Ultrasound in medicine \& biology 46, 518-543 (2020).

33. S. Turco, H. Wijkstra, M. Mischi, Mathematical models of contrast transport kinetics for cancer diagnostic imaging: a review. IEEE reviews in biomedical engineering 9, 121-147 (2016).

34. A. A. Lammertsma, S. P. Hume, Simplified reference tissue model for PET receptor studies. Neuroimage 4, 153-158 (1996).

35. P. Virtanen et al., SciPy 1.0: fundamental algorithms for scientific computing in Python. Nature methods 17, 261-272 (2020).

36. M. Lin, H. C. Lucas Jr, G. Shmueli, Research commentary - too big to fail: large samples and the p-value problem. Information Systems Research 24, 906-917 (2013).

37. M. Mischi, T. A. Kalker, E. H. Korsten, Contrast echocardiography for pulmonary blood volume quantification. IEEE transactions on ultrasonics, ferroelectrics, and frequency control 51, 1137-1147 (2004).

38. J. Rouwkema, A. Khademhosseini, Vascularization and angiogenesis in tissue engineering: beyond creating static networks. Trends in biotechnology 34, 733-745 (2016).

39. R. K. Jain, Determinants of tumor blood flow: a review. Cancer research 48, 2641-2658 (1988). 